\title{
Evaluación de atributos de crecimiento y desarrollo en genotipos de girasol asociados a distintos ambientes en tres regiones de Argentina
}

\author{
Arce de Caram, Gladis E. y Valentinúz, Oscar R. \\ Lugar de trabajo: Facultad de Ciencias Agrarias. UNNE. \\ E-mail: gladisarce@agr.unne.edu.ar
}

\begin{abstract}
Resumen
El cultivo de girasol (Helianthus annus L.) se siembra en la Argentina en un amplio rango de ambientes. Bajo la hipótesis de que la estabilidad productiva de genotipos de girasol está asociada a caracteres de desarrollo y crecimiento que mejoran la captura y uso de los recursos en los ambientes determinados por las regiones y fechas de siembra, se definieron los siguientes objetivos: i) caracterizar las relaciones existentes entre las variables climáticas que determinan la estación de crecimiento del girasol de cada región y el rendimiento, ii) analizar la interacción genotipo por ambiente; iii) evaluar caracteres de crecimiento y desarrollo en genotipos de girasol sembrados en las fechas óptimas y tardías en cada región y iv) establecer relaciones entre caracteres y la superioridad de genotipos de girasol. Se trabajó con información generada en experimentos conducidos en las regiones Pampeana, Litoral y Norte,en las localidades de Balcarce, Paraná y Sáenz Peña respectivamente, entre los años 2006 y 2008. Se compararon genotipos contrastantes combinados con dos fechas de siembra (temprana y tardía). Los resultados de este trabajo permitieron caracterizar climática y productivamente una diversidad de ambientes generados por las combinaciones entre localidades y fechas de siembra del cultivo. A su vez, la variabilidad en el rendimiento expresada por los genotipos en los distintos ambientes estuvo asociada en mayor medida a variables relacionadas con el desarrollo (ciclo, duración de subetapas), y en menor medida a variables relacionadas con el crecimiento (índice de área foliar, tasas de crecimiento en períodos críticos) y el rendimiento en grano y aceite.
\end{abstract}

Palabras clave: Genotipos de girasol, interacción genotipo- ambiente, fechas de siembra.

\begin{abstract}
Summary
Sunflower (Helianthus annus L.) production in Argentina is performed in a wide range of environments. Oil and grain yield stability of sunflower hybrids could be related to the ability to capture and use of resources (i.e., radiation, water, nutrients) among diversity of environments determined by regions and sowing date combinations. The objectives of this work were i) to identify relationships between climatic parameters and sunflower performance for a wide range of environments, ii) to analyze interation environment $\mathrm{x}$ hybrid iii) to quantify hybrid responses in crop growth and development under optimal and non-optimal sowing dates, and iv) to find associations between crop attributes and superiority (i.e., yield performance and stability) in sunflower hybrids. The study included data sets from experiments carried out across three regions covering temperate and sub-tropical environments. Thus, experiments performed during 2006-2008 growing seasons at three locations (Balcarce, Paraná y Sáenz Peña) with two sowing dates and hybrids combinations were analyzed.

A number of environments, determined by regions and sowing dates combinations, were characterized according to climatic and production variables. Environment $\mathrm{x}$ hybrid interaction for grain yield was primary associated to crop development (i.e., length of cycle and duration of vegetative and reproductive periods), and, in lower extend, to crop growth (leaf area index, crop growth rate during critical periods) and, oil and grain yield.
\end{abstract}

Key words: Sunflower, hybrids, sowing date, hibrid x environment interaction.

\section{INTRODUCCIÓN}

El girasol (Helianthus annuus L.) se siembra en Argentina desde el Chaco $\left(26^{\circ} \mathrm{S}\right)$ hasta el Sur de Buenos Aires $\left(39^{\circ} \mathrm{S}\right)$. Las principales zonas de producción nacional abarcan las provincias de Buenos Aires, La Pampa, Córdoba, Santa Fe, Entre Ríos, Santiago del Estero y Chaco (De la Vega, 2001).

El rendimiento potencial del cultivo por unidad de área se ubica en un rango que va desde aproximadamente 5000 a $6000 \mathrm{~kg}$ ha-1 en el sudeste de la región
Pampeana, en la localidad de Hilario Ascasubi hasta alrededor de $2300 \mathrm{~kg} \mathrm{ha}^{-1}$ en el noreste del país (Sadras, 2004).

Es así que los rendimientos muestran una variación considerable entre regiones, siendo las áreas subtropicales las de menor producción (Pascale y Damario, 1995).

Este cultivo experimenta variaciones interanuales tanto en rendimiento como en área sembrada. La Secretaría de Agricultura, Ganadería, Pesca y Ali- 
mentos de la Nación informó en la campaña agrícola 2007-2008 que la superficie sembrada con girasol se incrementó un $11,7 \%$ en relación al año anterior, lo que representó 2,7 millones de hectáreas. Este incremento en la superficie tuvo como factor principal el aumento del área sembrada en las provincias de Buenos Aires, Entre Ríos, La Pampa y Santa Fe, que permitió compensar el descenso de los rendimientos de otras provincias tales como Chaco y Santiago del Estero. Los rendimientos promedios en esta campaña en provincias con mayor tecnología de producción, superaron ampliamente al promedio nacional actual de $1800 \mathrm{~kg}$ ha $^{-1}$ (Asociación Argentina de Girasol, 2007).

La productividad de un cultivo está determinada por su potencial genético y el impacto del ambiente sobre su capacidad de crecimiento y partición de fotoasimilados entre partes cosechables y partes no cosechables (Gifford et al., 1984; Andrade et al., 1993).

El ciclo promedio del cultivo de girasol, comprende entre 100 y 150 días según genotipos, fechas de siembra, latitud y disponibilidad de agua y nutrientes (Díaz Zorita et al., 2003). Durante el crecimiento y desarrollo del cultivo, en la etapa de germinaciónemergencia, los factores más importantes son la humedad y temperatura de suelo. Si la temperatura de suelo al momento de la siembra es superior a $15^{\circ} \mathrm{C}$, el período se acorta, con temperaturas menores la emergencia se hace más lenta afectando el stand de plantas. En la etapa siguiente que corresponde al período comprendido entre la emisión del primer par de hojas verdaderas pares, y la aparición y expansión de la primera hoja impar alterna, la planta comienza a fotosintetizar y a acumular materia seca, el crecimiento foliar es muy lento. En siembras tempranas este periodo es prolongado, mientras que en las tardías se acorta sensiblemente. La transición entre la etapa vegetativa a reproductiva, está marcada por la diferenciación de los tejidos de la inflorescencia, temperaturas cercanas a $0^{\circ} \mathrm{C}$ pueden afectar el ápice generativo e impedir el desarrollo futuro del órgano floral (Díaz Zorita et al., 2003).

Durante la floración, que está comprendida entre la expansión de las flores liguladas y su marchitamiento, inmediatamente después de la fecundación de las últimas flores fértiles, comienza el periodo de llenado de fruto, que es el momento en que se acumulan materia seca, aceite y proteínas. El girasol acumula del $80 \%$ de su materia seca total antes de comenzar, el llenado de los granos. Esta etapa depende de la removilización de sustancias de reserva acumulada en los órganos vegetativos (principalmente tallos y hojas) hacia el capítulo, y de la actividad fotosintética de las hojas que permanezcan verdes después de la floración. Los fotosimilatos producidos después de la floración son particionados principalmente hacia los granos (Trápani y López Pereira, 2004).
Los diferentes componentes de rendimiento, se definen en diferentes etapas fenológicas del cultivo. El número de plantas comienza a determinarse a la siembra y finaliza luego de la emergencia de las plántulas, aproximadamente cuando estas alcanzan el estado de cuatro hojas verdaderas. El número de granos se determina durante una larga etapa, que comienza en la iniciación floral y el peso por grano comienza a determinarse aproximadamente en el estado de fin de floración y queda establecido en madurez fisiológica. Por último, el porcentaje de aceite, comienza a determinarse algunos días después que el peso por grano y generalmente queda establecido algunos días antes de la madurez fisiológica (Aguirrezábal, 2003).

Dado que los componentes del rendimiento se definen en diferentes estadíos fenológicos, las condiciones ambientales variables a lo largo del ciclo del cultivo, afectan el rendimiento a través de distintos componentes. La funcionalidad de hojas y raíces determina la capacidad del cultivo de capturar y utilizar los recursos del ambiente (radiación, agua, nutrientes). La estructura del cultivo es resultado de la distribución espacial de hojas y raíces (Díaz Zorita et al., 2003).

El contenido máximo de aceite está controlado genéticamente y se ha incrementado significativamente por mejoramiento genético (Sadras and Villalobos, 1994). La expresión de este carácter es influenciada por factores ambientales y prácticas agronómicas (Aguirrezábal y Pereyra, 1998). La concentración de aceite en el grano varía entre 48 y 54\%. Cuando se considera la concentración en el embrión, este porcentaje se ubica entre 65-70\% (López Pereira et al., 1999).

El peso del grano disminuye desde la periferia al centro del capítulo y la temperatura tiene un marcado efecto sobre el peso final del embrión, el componente mayor del grano. Además una mayor intercepción de radiación por la planta durante el llenado de grano tiene un efecto positivo sobre el peso del mismo (Dosio et al., 2000).

Muchos de los factores meteorológicos, edáficos y biológicos no son controlables en los cultivos extensivos de girasol. Sin embargo las variaciones de alguno de ellos son esperables y relativamente predecibles a una escala de tiempo semanal o mensual (temperatura y radiación solar incidente a lo largo de ciclo de cultivo), mientras que otros como la capacidad potencial del suelo para aportar nutrientes a las plantas, son caracterizables a partir de análisis específicos (Aguirrezábal et al., 1996).

Para lograr altos rendimientos, se necesitan: i) altas tasas de crecimiento, para lo cual se requieren altas radiaciones y temperaturas diurnas óptimas para el proceso fotosintético y ii) prolongadas duraciones de las etapas fenológicas, especialmente de aquellas más críticas para la determinación del rendimiento. 
En girasol, estas mayores duraciones se logran con bajas temperaturas (pero por encima de los niveles que dañan los tejidos). Esto es así, siempre y cuando el alargamiento del ciclo no ponga al cultivo en riesgo hacia el final de la estación de crecimiento ya que las condiciones climáticas y edáficas durante la etapa de secado y cosecha deben favorecer la recolección de prácticamente la totalidad de lo producido, sin pérdidas en la calidad del mismo (Trápani y López Pereira, 2004).

Los ambientes no sólo pueden definirse en términos geográficos, sino también por los factores bióticos y abióticos que influyen sobre el rendimiento del cultivo, entre los que se encuentran radiación, temperatura, estrés hídrico, etc. Las zonas ecológicas donde es factible la siembra de girasol se caracterizan por cambios en la duración del día, durante la estación de cultivo (primavera - verano), que se incrementa de norte a sur. La presencia de altas temperatura, en las regiones centro-nordeste del país constituye una de las limitantes más importantes para obtener altos rendimientos. Las deficiencias hídricas, constituyen otro factor que está presente en todas las regiones, y en mayor medida en los ambientes sub-húmedos y semiáridos del oeste de la región de cultivo (López Pereira y Trápani, 2004).

En los sistemas de producción el rendimiento es el resultado de múltiples interacciones, que varían de acuerdo a la región (López Pereira y Trápani, 2004). El cultivo se debe manejar de forma tal que los períodos más críticos para la determinación del rendimiento ocurran en el momento que las condiciones sean favorables para el cultivo (Andrade et al, 1996). Por ello la elección de la fecha de siembra es una estrategia de escape para evitar o disminuir el efecto de estrés abiótico y es utilizada en distintas zonas del país donde la siembra se inicia con la llegada de las precipitaciones al final de la primavera. Tanto los cultivares utilizados en cada zona, como las condiciones ambientales, de la estación de crecimiento y los sistemas de cultivos utilizados muestran una alta variación (Otegui y López Pereyra, 2003).

La relación entre la radiación y la temperatura, o cociente fototermal $(\mathrm{Q})$ en un ambiente dado es un índice que ha permitido explicar satisfactoriamente el rendimiento potencial de una especie como trigo principalmente (Fischer, 1985), también ha sido utilizada en girasol por Cantagallo (1997).

En este trabajo se estudiaron algunos factores ambientales que impactan sobre el rendimiento de diferentes híbridos (distinto ciclo y zona de adaptación) de girasol en las regiones Nordeste, Litoral y Pampeana con el objetivo de evaluar caracteres de crecimiento y desarrollo en híbridos de girasol sembrados en las fechas óptimas y tardías asociadas a cada región.

\section{MATERIALES Y MÉTODOS}

Este trabajo se realizó sobre una transecta de dirección noreste-sureste, desde los $26^{\circ}$ hasta los $37^{\circ}$ de latitud Sur, y desde $58^{\circ}$ a los $60^{\circ}$ de longitud oeste, en las regiones girasoleras Pampeana Litoral y Noreste. Esta última comprende a las provincias de Chaco, Formosa, centro y noreste de Santa Fe. El clima de esta región es subtropical con o sin estación seca. Su rasgo más saliente son las altas temperaturas y los veranos prolongados. Es común el déficit hídrico, cuyo efecto sobre el cultivo se ve normalmente agravado por las altas temperaturas. Los meses de julio hasta agosto son considerados los mejores para la siembra del girasol (Otegui y López Pereira, 2003).

La región Litoral comprende a la provincia de Entre Ríos. El clima es templado pampeano, cuyo rasgo característico es la escasa amplitud térmica anual. Tanto los veranos como los inviernos son moderados. En los meses de primavera y verano se registran las mayores precipitaciones, concentrándose entre octubre y abril el $79 \%$ del total de precipitaciones (Muller y Costa América, 2004).

La región Pampeana comprende al sur de la provincia de Buenos Aires, el clima es templado, con una marcada amplitud térmica anual y precipitaciones que disminuyen de este a oeste. Siembras muy tempranas aumentan el riesgo de soportar heladas o temperaturas cercanas a cero. Este fenómeno también puede presentarse en siembras tardías de la región.

Los experimentos fueron ajustados a un protocolo exclusivo para la obtención de datos estandarizados. Éstos incluyeron dos fechas de siembra, temprana y tardía y siete híbridos seleccionados por su ciclo, contenido en aceite, zona de adaptación (Tablas 1 y 2).

Tabla 1. Características de los diez ambientes. Fecha de siembra, ciclo agrícola y localidad.

\begin{tabular}{lccc}
\hline Ambiente & Ciclo Agrícola & Localidad & Fecha Siembra \\
\hline BA61 & $2006-07$ & Balcarce & $23-10-06$ \\
BA62 & $2006-07$ & Balcarce & $05-12-06$ \\
BA71 & $2007-08$ & Balcarce & $14-11-07$ \\
BA72 & $2007-08$ & Balcarce & $12-12-07$ \\
PA61 & $2006-07$ & Paraná & $02-11-06$ \\
PA62 & $2006-07$ & Paraná & $23-12-06$ \\
PA72 & $2007-08$ & Paraná & $18-12-07$ \\
SP61 & $2006-07$ & Sáenz Peña & $08-08-06$ \\
SP62 & $2006-07$ & Sáenz Peña & $27-10-06$ \\
SP72 & $2007-08$ & Sáenz Peña & $20-12-07$ \\
\hline
\end{tabular}

$\mathrm{BA}=$ Balcarce, $\mathrm{PA}=$ Paraná, $\mathrm{SP}=$ Sáenz Peña. 61= año 2006-07 y fecha siembra temprana; $62=2006-2007$ y fecha tardía.; 71 $=2007-08$ y fecha siembra temprana; $72=$ 2007-08 y siembra tardía. 
Tabla 2. Nombre comercial, empresa a la que pertenece, duración del ciclo y zona de adaptación de los siete híbridos de girasol.

\begin{tabular}{lcccc}
\hline Híbridos & Empresa & Duración Ciclo & $\begin{array}{c}\text { Color } \\
\text { Pericarpio }\end{array}$ & $\begin{array}{c}\text { Zona } \\
\text { Adaptación } \\
\text { (Argentina) }\end{array}$ \\
\hline AGROBEL 967 & Seminium & Corto/Intermedio & Negro & Amplia \\
DK 3820 & Monsanto & Medio-corto & Negro & Amplia \\
DK 3845 & Monsanto & Corto & Negro & Centro-Sur \\
DK 3940 & Monsanto & Medio & Estriado & Amplia \\
MG 2 & Dow Morgan & Intermedio & Estriado & Amplia \\
TRISOL 600 & Morgan & Intermedio & Estriado & Amplia \\
VDH 487 & Advanta & Intermedio & Negro & Amplia \\
\hline
\end{tabular}

Tabla 3. Datos de fecha de siembra; temperatura media diaria; temperatura máxima diaria, temperatura mínima diaria, radiación global, precipitaciones medias durante el ciclo de cultivo y abreviatura de los 10 ambientes de las tres localidades.

\begin{tabular}{lccccccr}
\hline Ambientes & Campaña & FS & $\begin{array}{c}\text { TMD } \\
\left({ }^{\circ} \mathbf{C}\right)\end{array}$ & $\begin{array}{c}\text { TMax } \\
\left({ }^{\circ} \mathbf{C}\right)\end{array}$ & $\begin{array}{c}\text { TMin } \\
\left({ }^{\circ} \mathbf{C}\right)\end{array}$ & $\begin{array}{c}\text { RG } \\
\left(\mathbf{M j}^{-2} \mathbf{m}^{-2} \mathbf{d}^{-1}\right)\end{array}$ & $\begin{array}{c}\text { Pr. } \\
(\mathbf{m m})\end{array}$ \\
\hline BA61 & $2006-07$ & $23-10-06$ & 18,7 & 27,6 & 13,2 & 2706,9 & 322,8 \\
BA62 & $2006-07$ & $05-12-06$ & 20,4 & 26,6 & 13,6 & 416,1 & 95,5 \\
BA71 & $2007-08$ & $14-11-07$ & 19,5 & 26,9 & 13,7 & 2184,8 & 282,1 \\
BA72 & $2007-08$ & $12-12-07$ & 20,4 & 26,4 & 15,0 & 2001,5 & 374,6 \\
PA61 & $2006-07$ & $02-11-06$ & 23,1 & 29,2 & 18,5 & 2685,0 & 919,0 \\
PA62 & $2006-07$ & $23-12-06$ & 22,7 & 28,0 & 18,3 & 2418,2 & 1175,6 \\
PA72 & $2007-08$ & $18-12-07$ & 24,1 & 29,7 & 18,3 & 1991,2 & 1865,3 \\
SP61 & $2006-07$ & $08-08-06$ & 23,5 & 30,5 & 16,3 & 3359,6 & 530,2 \\
SP62 & $2006-07$ & $27-10-06$ & 26,1 & 32,9 & 20,6 & 2997,4 & 495,4 \\
SP72 & $2007-08$ & $20-12-07$ & 24,2 & 32,2 & 19,9 & 2104,5 & 383,5 \\
\hline
\end{tabular}

FS $=$ Fecha de Siembra; TMD $=$ Temperatura Media Diaria; $\mathrm{TMax}=$ Temperatura Máxima Diaria; TMin= Temperatura Mínima Diaria, $\mathrm{RG}=\mathrm{Ra}$ diación Global; $\mathrm{Pr}=$ Precipitaciones; Ambientes son: $\mathrm{BA}=$ Balcarce, $\mathrm{PA}=$ Paraná, $\mathrm{SP}=$ Sáenz Peña. 61= año 2006-07 y fecha siembra temprana; 62= 2006-2007 y fecha tardía.; 71=2007-08 y fecha siembra temprana; 72= 2007-08 y siembra tardía.

Los ensayos se ejecutaron en las EEA del INTA ubicadas en las localidades de Balcarce $\left(37^{\circ} 45^{\prime} \mathrm{S}\right.$, $\left.58^{\circ} 18^{\prime} \mathrm{W}\right)$, Paraná $\left(31^{\circ} 50^{\prime} \mathrm{S}, 60^{\circ} 31^{\prime} \mathrm{W}\right)$ y Sáenz Peña $\left(26^{\circ} 52^{\prime} \mathrm{S}, 60^{\circ} 27^{\prime} \mathrm{W}\right)$. En cada una de ellas se utilizó un diseño en bloques completos al azar con tres repeticiones. Los tratamientos estuvieron representados por 7 híbridos (Tabla 2). El tamaño de las parcelas fue de 6 surcos por $8 \mathrm{~m}$ de largo cada uno, la distancia entre surcos fue de $0,70 \mathrm{~m}$ en Balcarce y Sáenz Peña y en Paraná $0,52 \mathrm{~m}$, para cada fecha de siembra. Cada experimento estuvo rodeado con una bordura importante compuesta por 4 surcos. La densidad poblacional fue de 50.000 plantas ha-1, lo que representa 3,5 plantas por metro lineal de surco.

En Balcarce y Sáenz Peña, las siembras fueron realizadas con la labranza necesaria para lograr una cama de siembra pareja para asegurar una buena implantación del cultivo.

En Paraná, se realizó la siembra sin labranza, es decir, sin movimiento del suelo. La siembra fue realizada manualmente en las tres localidades, también en el momento de la siembra se realizó un muestreo se suelo para conocer el estado nutricional del mismo y en caso de ser necesario se fertilizó según lo establecido en el protocolo; se determinó también el contenido de humedad en los primeros centímetros de suelo, para determinar la necesidad de riego.

Se llevó un registro meteorológico de las variables más importantes provisto por cada EEA de INTA. También se registró el desarrollo fenológico en cuatro plantas por parcela en intervalos regulares de tiempo, según la metodología de Schneiter y Miller (1981). Así se identificaron los estados vegetativos y reproductivos considerados más importantes para el cultivo.

Durante el ciclo del cultivo se realizaron aplicaciones preventivas con fungicidas e insecticidas y curativas en caso de ser necesario, para mantener los ensayos libres de malezas, plagas y enfermedades.

Las variables registradas fueron: 1) Fenología siguiendo la clave de Schneiter y Miller (1981), se registró la fecha de cada fase fenológica consideradas agronómicamente claves (emergencia, floración plena o R5.5, fin de floración o R6, y madurez fisiológica o R9), en 3 plantas por parcela y dos veces por semana, el área foliar en R5.5, valor máximo IAF (Pereyra et al., 1982), evolución de la radiación interceptada de acuerdo a Dosio et al. (2000), al mediodía solar ( \pm 1 hora) con un sensor lineal cuántico (LI-191SB, LICOR, Lincoln, NE, USA). La fracción de radiación solar interceptada se calculó como [1-(Rb/Rs)] según Gallo y Daughtry (1986), donde $\mathrm{Rb}$ es la radiación medida en la última hoja verde inferior de las plantas y Rs es la radiación incidente sobre la canopia. Para la determinación de la biomasa aérea seca en R5.5, se cortaron 3 plantas por parcela, se las particionó y se registró el número total de hojas (NTH). La tasa de crecimiento del cultivo desde emergencia a antesis (R5.5), como cociente entre la materia seca acumu- 
Tabla 4. Variables utilizadas, para estudiar el efecto de los ambientes en las tres regiones y sus abreviaturas.

\begin{tabular}{ll}
\hline VARIABLE & ABREVIATURAS \\
\hline $\begin{array}{l}\text { Tasa de crecimiento por planta materia } \\
\text { seca acumulada desde emergencia } \\
\text { plena floración (R5.5) por número de } \\
\text { días transcurrido. }\end{array}$ & TCCEaR5.5 \\
$\begin{array}{l}\text { Tiempo térmico acumulado desde } \\
\text { emergencia hasta madurez fisiológica }\end{array}$ & TTEaMF \\
$\begin{array}{l}\text { Tiempo térmico acumulado desde plena } \\
\text { floración hasta madurez fisiológica }\end{array}$ & TTR5.5aMF \\
$\begin{array}{l}\text { Número total de hojas en plena } \\
\text { floración }\end{array}$ & NTH \\
$\begin{array}{l}\text { Índice de Área Foliar a plena floración } \\
\text { Días desde emergencia hasta madurez } \\
\text { fisiológica }\end{array}$ & IAF \\
$\begin{array}{l}\text { Temperatura Máxima promedio durante } \\
\text { el ciclo del cultivo }\end{array}$ & TMax \\
$\begin{array}{l}\text { Temperatura Mínima promedio durante } \\
\text { el ciclo del cultivo }\end{array}$ & TMin \\
Rendimiento en (kg ha-1) & \\
Porcentaje de aceite (\%) & RTO \\
\hline
\end{tabular}

lada desde emergencia hasta floración y número de días transcurridos (Gardner et al., 1985).

A partir de los datos meteorológicos y fenológicos del cultivo, siguiendo el criterio de los ensayos preliminares, se utilizaron los dos sub-períodos i) emergencia a plena floración y ii) plena floración a madurez fisiológica. Las variables calculadas fueron: a) tiempo térmico acumulado desde emergencia hasta madurez fisiológica (TTEaMF), b) tiempo térmico acumulado entre antesis y madurez fisiológica (TTR5.5aMF), c) tasa de crecimiento del cultivo desde emergencia hasta plena floración (TCCE a R5.5), d) número total hojas (NTH), e) índice de área foliar (IAF), f) Rendimiento en grano (RTO), g) Porcentaje de aceite del grano $(\% \mathrm{~A}), \mathrm{h})$ Peso de 1000 granos (Tabla 4).

Los componentes del rendimiento se evaluaron cortando los capítulos de los surcos centrales de cada parcela. Una vez secados al aire, se trillaron registrándose el peso y contenido de humedad. De una muestra de $500 \mathrm{~g}$ se determinó el porcentaje de aceite y el peso de los 1000 granos (Aguirrezábal et al., 1996).

La Tabla 3 muestra el resumen de las variables meteorológicas, fechas de siembras, año de realización y nomenclatura que caracteriza a los ambientes.

Se realizaron análisis de varianza para las variables rendimiento en grano $\left(\mathrm{kg} \mathrm{ha}^{-1}\right)$ y porcentaje de aceite en el grano $(\% \mathrm{~A})$ y pruebas multivariantes entre los híbridos, variables meteorológicas, y ambientes a fin de realizar un análisis descriptivo y/o lograr inferencias a partir de los datos obtenidos (InfoStat, versión 2011)

\section{RESULTADOS}

Los experimentos analizados, corresponden a las localidades Balcarce, Paraná y Sáenz Peña, representativas de las regiones girasoleras Pampeana, Litoral y Noreste durante dos ciclos agrícolas (2006-2007 y 2007-2008.)

En cada localidad, se utilizó un grupo de 7 híbridos seleccionados en base a características contrastante en términos de ciclo, zona de adaptación, material genético, etc. y sembrados en dos fechas de siembra, coincidente con los extremos de la ventana de siembra para cada localidad (Tabla 5).

En Sáenz Peña, si bien la siembra fue posterior, a la ocurrencia de precipitaciones, la presencia de años con sequía, hizo del riego una práctica necesaria. La restricción por disponibilidad hídrica inicial, en la zona norte, puede llegar a ubicar la floración, y el llenado de los granos (etapas críticas), en cultivo de maíz y girasol, en un período de muy elevadas temperaturas durante el verano, con los efectos negativos que produce (Otegui y López Pereira, 2003).

Se observó una amplia variabilidad en el rendimiento de grano y porcentaje de aceite, en los distintos de ambientes. En general, los rendimientos de los ensayos de Balcarce, superaron a los de Paraná y Sáenz Peña. En el análisis de la varianza del rendimiento de grano, se pudo ver el efecto de los ambientes (representados por el año y la fecha de siembra), como también de los híbridos y la interacción híbrido por ambiente (Tabla 6).

Tabla 5. Rendimiento de grano $\left(\mathrm{kg} \mathrm{ha}^{-1}\right)$ y contenido de aceite (\%A), diez ambientes y las dos fechas de siembra.

\begin{tabular}{cccc}
\hline AMBIENTE & $\begin{array}{c}\text { FECHA } \\
\text { SIEMBRA }\end{array}$ & $\begin{array}{c}\text { RTO } \\
\text { (kg ha-1) }\end{array}$ & $\begin{array}{c}\text { A } \\
\text { (\%) }\end{array}$ \\
\hline BA61 & $23-10-06$ & 3221 & 47,5 \\
BA62 & $05-12-06$ & 2868 & 45,7 \\
BA71 & $14-11-07$ & 4027 & 51,1 \\
BA72 & $12-12-07$ & 2029 & 42,0 \\
PA61 & $02-11-06$ & 1576 & 37,3 \\
PA62 & $23-12-06$ & 1300 & 46,6 \\
PA72 & $18-12-07$ & 3615 & 44,4 \\
SP61 & $08-08-06$ & 1746 & 50,4 \\
SP62 & $27-10-06$ & 2180 & 51,3 \\
SP72 & $20-12-07$ & 1842 & 42,7 \\
\hline
\end{tabular}

$\mathrm{BA}=$ Balcarce, $\mathrm{PA}=$ Paraná, $\mathrm{SP}=$ Sáenz Peña. $61=$ año 2006-07 y fecha siembra temprana; $62=2006-2007$ y fecha tardía.; $71=2007-08$ y fecha siembra temprana; $72=$ 2007-08 y siembra tardía. 
Tabla 6. Análisis de la varianza del rendimiento de grano $\left(\mathrm{kg} \mathrm{ha}^{-1}\right)$, en las campañas 2006-2007 y 2007-2008, de los 10 ambientes.

\begin{tabular}{lrrrrrr}
\hline F.V. & \multicolumn{1}{c}{ SC } & \multicolumn{1}{c}{ gl } & \multicolumn{1}{c}{ CM } & \multicolumn{1}{c}{ F } & p-valor & (Error) \\
\hline Ambiente & 3608,74 & 9 & 400,97 & 63,09 & $<0,0001$ & (Ambiente $>$ rep) \\
Hibrido & 1041,39 & 6 & 173,56 & 60,31 & $<0,0001$ & \\
Ambiente>rep & 152,54 & 24 & 6,36 & 2,21 & 0,0029 & \\
Hibrido*Ambiente & 848,58 & 54 & 15,71 & 5,46 & $<0,0001$ & \\
Error & 328,10 & 114 & 2,88 & & & \\
\hline
\end{tabular}

Posteriormente se realizó un análisis de componentes principales (CP), donde cada componente es una combinación de las variables originales. La correlación entre cada componente y las variables originales establece cuales de estas variables
Tabla 7. Rendimiento de grano $\left(\mathrm{kg} \mathrm{ha}^{-1}\right)$, contenido de aceite $(\% \mathrm{~A})$, y variables de crecimiento, desarrollo, en dos subperíodos, respecto de las componentes principales 1 y 2 , y porcentaje de la variancia explicada por cada componente, abreviaturas según Tabla 8.

\begin{tabular}{lcc}
\hline \multirow{2}{*}{ VARIABLES } & \multicolumn{2}{c}{ COEF. CORRELACION } \\
\cline { 2 - 3 } RTO & CP1 & CP2 \\
\hline \%A & $-0,59$ & $\mathbf{0 , 7 4}$ \\
TTR5.5-MF & $\mathbf{0 , 8 2}$ & 0,32 \\
TTE-MF & $\mathbf{0 , 7 9}$ & 0,18 \\
TCCE-R5.5 & $\mathbf{0 , 8 8}$ & 0,30 \\
NTH & 0,57 & 0,18 \\
IAF & 0,69 & $\mathbf{0 , 6 6}$ \\
P1000 & $-0,62$ & $\mathbf{0 , 6 6}$ \\
\hline \% VARIAC. EXPLICADA & $\mathbf{0 , 7 2}$ & 0,55 \\
\hline
\end{tabular}

El rendimiento de grano fue máximo en el ambiente Balcarce, siembra temprana, año 2007-2008, y mínimo en el ambiente de Paraná siembra tardía, año 2006-2007, con 4027 y $1300 \mathrm{~kg} \mathrm{ha}^{-1}$ respectivamente (Tabla 5).

En cuanto al contenido de aceite fueron altamente significativos los ambientes, híbridos y la interacción entre ambos. La localidad de Sáenz Peña fue la de mayor porcentaje promedio de aceite $48,1 \%$, seguida por Balcarce $46,6 \%$ de aceite y luego Paraná con 43,0 $\%$ de aceite (Tabla 5).

Estos resultados concuerdan con lo expresado por Aguirrezábal y Pereyra (1998) en que el porcentaje de aceite producido por un mismo cultivar puede presentar grandes variaciones entre años, regiones y fechas de siembra. Para un mismo híbrido sembrado en distintos ambientes (aún en la misma región en diferentes años) posee mayores variaciones que las que se producen entre híbridos de alto y bajo porcentaje de aceite. En consecuencia, todo factor ambiental que afecte la fotosíntesis durante el llenado de los granos o el transporte de carbohidratos a los mismos durante esta corta etapa es potencialmente capaz de afectar la acumulación del aceite en los frutos (Aguirrezábal y Pereyra, 1998). tienen mayor incidencia en cada componente principal y si su efecto es positivo o negativo.

Las respuestas de un conjunto de híbridos proveen una caracterización indirecta del ambiente. El análisis multivariante, realizado para evaluar cuáles fueron las variables de crecimiento y desarrollo que caracterizan cada uno de los ambientes estudiados indicó que el $77 \%$ de la variabilidad total fue explicada por los primeros dos componentes principales (Tabla 7, Figura 1). La CP1, que es la componente que explica por sí sola el $52 \%$ de la variabilidad total, separa los ambientes, a la izquierda los de la región Nordeste y a la derecha los de las regiones Pampeana y Litoral. Los vectores que son las variables de crecimiento y desarrollo del cultivo, y posee mayor peso en la CP1, corresponden al tiempo térmico en los dos subperíodos (TTR5.5aMF y TTEaMF), porcentaje de aceite $(\% \mathrm{~A})$ y una correlación negativa con el peso de mil semillas y el IAF. La duración de estas etapas será generalmente menor con temperaturas y radiación, altas, y días largos. Esto tiene consecuencias sobre el número final de hojas, que fue menor (Trápani y López Pereira, 2004). Estas variables caracterizan a los ambientes de Sáenz Peña (SP61 y SP62) en la campaña 2006-2007 en ambas fechas de siembra.

La CP2 que representa solo el $25 \%$ de la variabilidad total está asociada a las localidades de Paraná

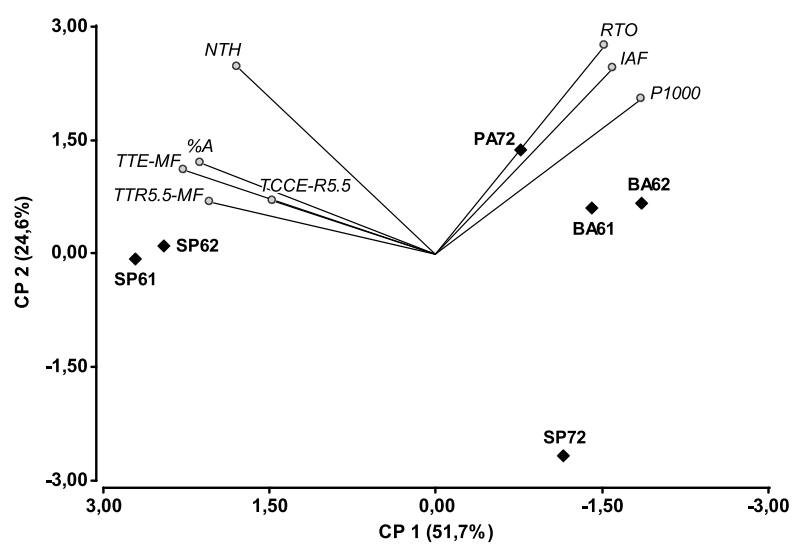

Figura 1. Biplots del 1er y 2do componente principal de los ambientes, y las variables de crecimiento y desarrollo, en las campañas 2006-2007; 2007-2008, los ambientes representados por rombos negros y las variables por vectores desde el origen. 
y Balcarce (PA72, BA61 y BA62), y corresponde a las variables rendimiento de grano, índice de área foliar, y peso de mil semillas. (Figura 1, línea envolvente puenteada) Como la producción de biomasa del cultivo depende de la cantidad de energía lumínica que interceptan sus hojas determinada por su índice de IAF y de la eficiencia de uso esa energía, a medida que aumenta la intercepción de radiación (área foliar verde) durante el llenado de grano se produce un efecto positivo por prolongar la duración de esta etapa como por mejorar la tasa de acumulación de peso en el grano- granos más pesado (Díaz Zorita et al., 2003). El ambiente SP72, Sáenz Peña ciclo 20072008 en siembra tardía, se mostró como un ambiente específico, que no se asocia a ninguna de las variables utilizadas.

\section{DISCUSIÓN}

Los sitios fueron caracterizados por radiación global incidente, temperaturas medias, valores de coeficiente fototérmico y adversidades desde siembra hasta madurez fisiológica.

Los ambientes de siembra temprana y tardía en las tres localidades presentaron fuertes diferencias en sus efectos sobre los patrones de comportamiento de los híbridos evaluados. Los resultados obtenidos concuerdan en que atrasos en la fecha de siembra expone a la planta a temperaturas altas y baja radiación durante el llenado del grano, que afectan el peso y la calidad (Otegui y López Pereyra, 2003).

Andrade (1995) ha establecido que la caída de los niveles de radiación y las bajas temperaturas durante el período crítico período de llenado del grano a fines del verano, en siembras tardías en Balcarce, determinan que el rendimiento se reduzca en aproximadamente un $35 \%$. Esta respuesta se debería a que en girasol la tasa de llenado de grano, es afectada por la capacidad fotosintética de la planta durante el llenado de los granos, especialmente aquellos granos ubicados en el centro del capítulo (Andrade y Ferreiro, 1996). También la duración de la etapa de llenado de grano, es afectada por modificaciones en la cantidad de radiación interceptada por planta (Dosio et al. 1997), y por altas temperaturas del capítulo durante este período crítico acortan la duración del mismo y reducen el peso del grano (Ploschuk y Hall, 1995). En Sáenz Peña el atraso en la fecha de siembra como lo expresara Mercau (2010), para evitar la exposición a heladas tardías, aumenta la exposición del llenado de los granos a temperaturas altas que reducen el rendimiento, por la presencia de altas temperatura, que reducen el peso final del grano, por reducir la duración del período de llenado y Rondanini (2006), en que altas temperaturas en el llenado no solo reducen el peso de los granos, sino también su porcentaje de grasa.
En la evaluación de los caracteres de crecimiento y desarrollo que podrían estar asociados a cada uno de los ambiente generados (Figura 1), se observó que los ambientes de la región Noreste se separan de los de las otras dos regiones y que las variables de crecimiento y desarrollo asociadas a la localidad de Sáenz Peña en ambas fechas de siembra (SP61 y SP62) fueron las relacionadas con la generación de la parte aérea y el tiempo térmico entre emergencia y floración y entre floración y madurez. En tanto, Balcarce y Paraná (Figura 1) se caracterizaron por poseer rendimientos más altos que estuvieron asociados al índice de área foliar y peso de grano. Estas observaciones son coherentes con las realizadas por otros autores, que han encontrado asociación positiva entre rendimiento y duración del área foliar durante el llenado y entre porcentaje o rendimiento de aceite y radiación interceptada durante el llenado (Andrade y Ferreiro, 1996; Dosio, 1998).

\section{CONCLUSIONES}

En el análisis de las relaciones entre caracteres de crecimiento y desarrollo del cultivo, y la respuesta de los híbridos de girasol presentaron variaciones en los ambientes confirmando que el contenido promedio de aceite de los híbridos en Sáenz Peña fue mayor que en las otras localidades. Las diferencia entre los bajos rendimientos de grano y porcentaje de aceite mayor en Sáenz Peña, estarían asociadas a las mayores temperaturas registradas durante la fase de llenado de granos, que constituye uno de los factores ambientales responsables de las diferencias entre las regiones.

Los resultados de este trabajo, permitieron caracterizar los ambientes en estudio correspondientes a las regiones Pampeana, Litoral y Norte, no solo las variables asociadas con el crecimiento y desarrollo, sino también las variables meteorológicas que definen la estación de crecimiento del cultivo y su rendimiento.

\section{BIBLIOGRAFIA}

Aguirrezábal, L.A.N, y Pereyra, V.R. 1998. Girasol. En: Aguirrezábal, L.A.N. y Andrade, F.H. Eds. "Calidad de productos agrícolas. Bases ecofisiológicas, genéticas y de manejo agronómico". Edición de la Facultad de Ciencias Agrarias (UNMdP) y de la Estación Experimental Balcarce del Instituto Nacional de Tecnología Agropecuaria, pp.139-192.

Aguirrezábal, L.A.N., Lavaud, Y., Dosio, G.A.A., Izquierdo, N.G., Andrade, F.H. and González, L.M. 2003. Intercepted solar radiation effect during seed filling determines sunflower weight per seed and oil concentration. Crop Sci. 43. Pp 152-161.

Aguirrezábal, L.A.N., Orioli, G.A., Hernández L.F., Pereyra V.R, Miravé, J.P., 1996. Girasol: aspectos fisiológicos 
que determinan el rendimiento. Editorial Unidad Integrada Balcarce (Balcarce, Argentina). 127 pp.

Andrade F.H., Uhart, S.A. and Cirilo, A. G. 1993. Temperature affects radiation use efficiency in maize. Field Crops Res. 32:17-25.

Andrade, F.H. y Ferreiro, M. 1996. Reproductive gowth of maize, sunflower and soybean at different source levels during grain filling. Field Crop Res. 48: 155-165.

Cantagallo, J. E., Chimenti, C. A., Hall, A. J. 1997. Number of seeds per unit area in sunflower correlates well with a photothermal quotient. Crop Sci. 37, 1780-1786.

De La Vega, A. 2001. Interacción genotipo por ambiente en girasol (Helianthus annus L.) megambientes, selección indirecta y bases fisiológicas de la adaptación a ambientes subtropicales. Tesis doctoral. Universidad de Buenos Aires.

Díaz Zorita, M., Duarte, G. y Plante, E. 2003. Guía para la producción de girasol en siembra directa, Monsanto, Buenos Aires, pp 29-37. Asociación Argentina de Girasol. ASAGIR .

Dosio, G.A.A., Aguirrezábal, L.A.N., Andrade, F.H., and Pereyra, V. R. 2000. Solar radiation intercepted during seed filling and oil production in two sunflower hybrids. Crop Science 40(6): 1637-1640.

Dosio, G.A.A., González, L.M., Pereyra, V.R. y Aguirrezábal L.A.N. 1998. Efecto de cortos períodos de reducción de la radiación incidente sobre los componentes del rendimiento en aceite en plantas de girasol. In Reunión Nacional de Fisiología Vegetal, 22o, Mar del Plata, Argentina, 162-163 pp.

Fischer R. A. 1985. Number of kernels in wheat crops and the influence of solar radiation and temperature. J. Agric. Sci. 105: $447-461$.

Gadner, F. P.; Pearce, R. B. and Mitchel, R. L. 1985. Physiology of crop plants. Iowa State University Press. USA. $327 p$.

Gallo, K.P. and C.S.T. Daughtry, 1986. Techniques for measuring intercepted and absorbed photosynthetically active radiation in corn canopies. Agronomy Journal. 78:752-756.

Gifford, R.M.; Thorne, J.H.; Hitz, W.D. and Giaquinta, R.T. 1984 Crop productivity and assimilate partitioning. Science, 225:801- 808.

InfoStat versión 2011. Di Rienz o J.A., Casanoves F., Balzarini M.G., Gonzalez L., Tablada M., Robledo C.W. Grupo InfoStat, FCA, Universidad Nacional de Córdoba, Argentina. URL http://www.infostat.com.ar.

López Pereira, M, Trapani, N., Sadras, V.O., 1999. Genetic improvement of sunflower in Argentina between 1930 and 1995. II. Phenological development, growth and source-sink relationship. Field Crops Res. 63, 247-254.

López Pereira, M. y Trápani, N. 2004. Capítulo 3: Importancia de Factores Abióticos que Limitan el rendimiento y la Calidad del Girasol. En: "El cultivo de girasol en siembra directa". Argentina. $1^{\circ}$ Ed. Buenos Aires. Monsanto.
Mercau, J. L., Sadras, V. O., Satorre, E. H., Messina, C., Balbi, C., Uribelarrea, M., and Hall, A. J. 2001. Onfarm assessment of regional and seasonal variation in sunflower yield in Argentina. Agricultural Systems 67: 83-103.

Müller, R. E. y Castro Almería, A. 2004. Modelo de producción en el litoral, Provincia de Entre Ríos. Capítulo 18. En El cultivo del girasol en siembra directa. Duarte, G. et al, 2004. $1^{\circ}$. Ed. Buenos Aires. Monsanto. 208 pp.

Otegui, M.E. y López Pereyra, M. 2003. Fecha de siembra. Capítulo 12. En: Producción de Cultivos de Grano. Bases funcionales para su manejo. (Ed. Pascale, A.J.) Editorial de la Facultad de Agronomía. Universidad de Buenos Aires.783 pp.

Pascale A. y Damario E. 2005. Bioclimatología Agrícola y Agroclimatología. Editorial de la Facultad de Agronomía. Universidad de Buenos Aires. 576 pp.

Pereyra V.R., Farizo C.L., Cardinali F.J. y Orioli G.A. 1982. Estimation of leaf area on sunflower plants. Proceedings Tenth International Sunflower Conference, Surfers Paradise, Australia. pp. 21-23.

Ploschuk, E.L., and A.J. Hall. 1995. Capitulum position in sunflower affects grain temperature and duration of grain filling. Field Crops Res. 44:111-117.

Rondanini, D. 2006. Efectos de breves periodos de altas temperaturas durante el llenado sobre la dinámica de crecimiento del fruto y la acumulación y calidad del aceite en girasol (Helianthus annuus L.). Ph.D. Thesis. Universidad de Buenos Aires, p. 209.

Sadras, V. O. 2004. Los máximos rendimientos. Capítulo 10. Ciclo ontogénico, dinámica del desarrollo y generación del rendimiento y la calidad en girasol. En Producción de Cultivos de Grano. Bases Fisiológicas para su manejo. (Ed. Pascale, A.J.) Editorial de la Facultad de Agronomía. Universidad de Buenos Aires.783 pp.

Sadras, V.O. y Villalobos, F.J. 1994. "Physiological characteristics related to yield improvement in sunflower (Helianthus annuus L.)". In: Genetic improvement of Field Crops (Gustavo A. Slafer ed.). Marcel Dekker, Inc. New York-Hong Kong, pp. 287-319.

Sheneiter, A.A. and J.F. Miller. 1981. Description of sunflower growth stages. Crop.Sci. 21: 901-903

Trápani, N. y López Pereira. 2004. Bases ecofisiológicas para la producción de girasol. En: Díaz-Zorita, M. y Duarte, G.A. Editores. Guía para la producción de girasol en siembra directa, Monsanto, Buenos Aires, pp2937. Argentina.

Trápani, N., Pereira, M. L., Sadras, V. O. y Hall, A. J. 2003. Ciclo ontogénico, dinámica del desarrollo y generación del rendimiento y la calidad en girasol. pp. 203-241. En: E. Satorre, R. Benech Arnold, G. A. Slafer, E. de la Fuente, D. Miralles, M. E. Otegui, y R Savin (eds). Producción de cultivos de granos. Bases funcionales para su manejo. Facultad de Agronomía. Buenos Aires. 